\title{
Fetal adenocarcinoma - a rare variant of lung adenocarcinoma with good prognosis
}

\author{
Jolanta Winek ${ }^{1}$, Paweł Caban ${ }^{2}$, Jacek Zych ${ }^{1}$, Renata Langfort ${ }^{3}$, \\ Barbara Burakowska ${ }^{4}$, Kazimierz Roszkowski-Śliż ${ }^{1}$
}

Fetal adenocarcinoma (FA) is a rare variant of lung adenocarcinoma with a good prognosis which represents approximately $0.5 \%$ of all primary lung neoplasms. The tumour has nonciliated tubules with morules in the lumen resembling fetal lung at 10 to 16 weeks of gestation. FA typically occurs in young patients $75-80 \%$ of whom are tobacco smokers. FA occurs in low- and high-grade forms. Surgical procedure is usually necessary to obtain final diagnosis and a complete surgical resection is the standard treatment. The 5-year survival rate for FA is about $75-80 \%$. We report a case of 32-year-old male, a smoker, who had an incomplete regression of parenchymal consolidations after antibiotic treatment, which led to further diagnostics. An intravenous tumour in one of the pulmonary veins with concomitant development of collateral circulation and pulmonary venous infarction were additional diagnostic problems. Based on the intraoperative microscopic examination, fetal adenocarcinoma was diagnosed. An upper right bilobectomy with intrapericardial ligation of the right superior pulmonary vein was performed as radical treatment. At the 9-month follow-up stage, there has been no evidence of disease recurrence.

NOWOTWORY J Oncol 2016; 66, 5: 381-385

Key words: fetal adenocarcinoma, pulmonary blastoma, lung cancer, prognosis

\section{Introduction}

Fetal adenocarcinoma (FA) occurs very rarely, accounting for only about $0.1-0.5 \%$ of all primary lung neoplasms [1, 2]. Its architecture is made up of tubules consisting of nonciliated cells rich in glycogen, resembling fetal lungs at 10 to 16 weeks of gestation [2,3]. Within the lumen of the tubules, there are the so-called morules made up of the cells with squamous differentiation. The presence of characteristic extranuclear vacuoles, rich in glycogen, makes adenocarcinoma resemble uteral mucosa in the secretion phase [4]. The specific feature of this carcinoma is the presence of argyrophil cells with neurosecretion activity [5, 6].

In 1982 Kradin et al. described the "pulmonary form of blastoma" containing in its architecture, argyrophil cells without sarcomatous features defining it as an endoder- mal lung tumour which resembles a fetal lung. Koss et al., analysing 52 lung blastoma cases distinguished its form lacking sarcomatous features, which later on was named a well-differentiated fetal adenocarcinoma. Its idiosyncratic feature, differentiating it from other blastoma forms, was a much better prognosis. According to the WHO tumour classification, since 1999 fetal adenocarcinoma has been considered as a histological variant of adenocarcinoma $[2,7]$.

Within a group of fetal adenocarcinomas, two groups of tumours have been distinguished: a well-differentiated form with a low-grade malignancy and a poorly-differentiated form with a high-grade malignancy. Fetal adenocarcinoma with a low-grade malignancy usually affects young people, mostly women with a moderate cigarette smoking history. The histopathological evaluation of this type of cancer re-

${ }_{1}^{1}{ }^{\text {rd }}$ Department of Lung Diseases, Institute of Tuberculosis and Lung Diseases, Warszawa, Poland ${ }^{2}$ Department of Chest Surgery, Institute of Tuberculosis and Lung Diseases, Warszawa, Poland ${ }^{3}$ Department of Pathomorphology, Institute of Tuberculosis and Lung Diseases, Warszawa, Poland ${ }^{4}$ Department of Radiology, Institute of Tuberculosis and Lung Diseases, Warszawa, Poland 
veals a small atypia of cellular nuclei and a tendency to create morules, as opposed to a less differentiated form. The disease is usually diagnosed at stage I and the mortality rate is about $10 \%$. The form with a high-grade malignancy usually affects patients a few decades later, concerning men with a long cigarette smoking history more often. The microscopic examination reveals a generalised disorganisation of glands, necrosis, cellular atypia and morules are quite rare. This tumour resembles more a typical lung adenocarcinoma and a component of other morphological subtypes of adenoma is more frequently found. This disease tends to be diagnosed at a more advanced stage, which results in a higher mortality rate $[8,9]$.

Although the pathogenesis of fetal adenocarcinoma remains unclear, in patients with a low malignancy degree form, the mutation of the $\mathrm{p} 53$ with the $\mathrm{p} 53$ protein hyperexpression related to this mutation was found. Nakatani et al. also detected some incorrect nuclear-cytoplasmatic $\beta$-catenin expression, which led them to a hypothesis that $\beta$-catenin mutation is involved in the proliferation of epithelial cells and the creation of morules $[8,10,11]$. The observation of children suffering from fetal adenocarcinoma show that this can be the rare form of DICER1 syndrome connected with somatic mutation or the mutation from the line of germinal cells [12]. Fetal adenocarcinoma with a high malignancy degree is not strongly connected with any genetic mutation.

The prognosis of patients with fetal adenocarcinoma, in comparison with more frequent forms of adenocarcinoma or fetal carcinoma, is good. It depends, first of all, on the grade of malignancy and the advancement stage of the cancer. In a group of patients with fetal adenocarcinoma with a low malignancy degree, the 5 -year survival rate is $75-80 \%$, whilst in a group of patients in the stage I of advancement it reaches even $90 \%[1,6-8,10]$. Poor prognosis is associated with the presence mediastinal lymphadenopathy, initial metastases and disease recurrence after treatment [13].

In the patients with a diagnose of a poorly differentiated form of this tumour, with a high-grade of malignancy, the prognosis depends also on morphological components of the adenocarcinoma which are present in the architecture of fetal adenocarcinoma [5].

This article presents the case of a young male patient, a cigarette smoker, in whose case the diagnostics of resistant parenchymal lesions in the lungs after an infection led to a diagnosis of fetal adenocarcinoma.

\section{Case report}

A 32-year-old male with a cigarette smoking history (7 smoking pack-years) was referred to the Institute of Tuberculosis and Lung Diseases with a suspicion of staphylococcal pneumonia. The disease began one month and a half before the admission to the Institute with the following symptoms: high fever, cough, haemoptysis and a strong pain in the right side of the chest. The patient was admitted to his regional hospital. Additional examinations revealed increased inflammation markers, such as leucocytosis - 11 thousand/ $\mu \mathrm{L}$ with an increased neutrophil count (8.49 thousand $/ \mu \mathrm{L}$ and increased CRP levels $172.59 \mathrm{mg} / \mathrm{L}$. The chest X-ray revealed diffuse opacities in the right upper lobe with a right hilum enlargement and a pleural reaction. Treatment with amoxicillin with clavulanic acid was initiated, resulting in an alleviation of high fever and pain within a few days. Additional follow-up tests revealed the normalisation of leucocytosis and a decrease of CRP levels ( $48.81 \mathrm{mg} / \mathrm{L}$ ). The patient was discharged home with the recommendation to continue antibiotics and perform a follow-up chest X-ray in two weeks.

The follow-up chest $X$ ray revealed a $44 \times 30 \mathrm{~mm}$ tumor in the right upper lobe with concomitant atelectasis. To extend the diagnosis a chest computed tomography was performed. In the right upper lobe it revealed the consolidated parenchymal lesions with irregular margins abutting the parietal pleura and oblique interlobar fissure with a visible air bronchogram, surrounded by ground glass opacities. Moreover, in segment 3 , some nodular tumour-like lesions were detected with contrast enhancement up to 57 Hounsfield Units (HU) with an enlargement of the lymph nodes of the right hilum. The largest tumour-like lesion was $44 \times 33 \mathrm{~mm}$, whilst the smaller ones were about $10 \mathrm{~mm}$. On the basis of the CT scan of the chest, a suspicion of staphylococcal pneumonia was made and the patient was urgently referred to the Institute of Tuberculosis.

Upon admission, the patient was in a good general condition, with some chest discomfort during deep breathing and a slight cough. Additional lab tests were unremarkable: the inflammation markers were no longer elevated and the D-dimer and LDH serum levels were normal. The chest X-ray revealed inhomogeneous patchy parenchymal densities in the right upper lobe with enlargement of the right hilum caused by a round lesion with a diameter of $18 \mathrm{~mm}$ (Fig. 1). During the consultations of the submitted chest CT scans with radiology specialists, it was found that the abovedescribed spherical mass filled the lumen of the superior pulmonary vein and the confluent densities correspond to pulmonary infraction. The echocardiography did not reveal any right ventricular overload or pulmonary hypertension. However, in the area of the right pulmonary venous drainage, it revealed some additional irregular echoes with a diameter up to $40 \mathrm{~mm}$, which were likely to press/infiltrate the right pulmonary veins and pericardium. Anticoagulants (LMWH) were introduced (a history of haemoptysis) in gradually increasing doses. With regards to the fact that the degree of intraluminal contrast filling in the submitted CT scan did not allow for a reliable evaluation of the relationship between the nodular-tumour-like lesions and the vessels, the examination was repeated with a angio-CT option. In the 


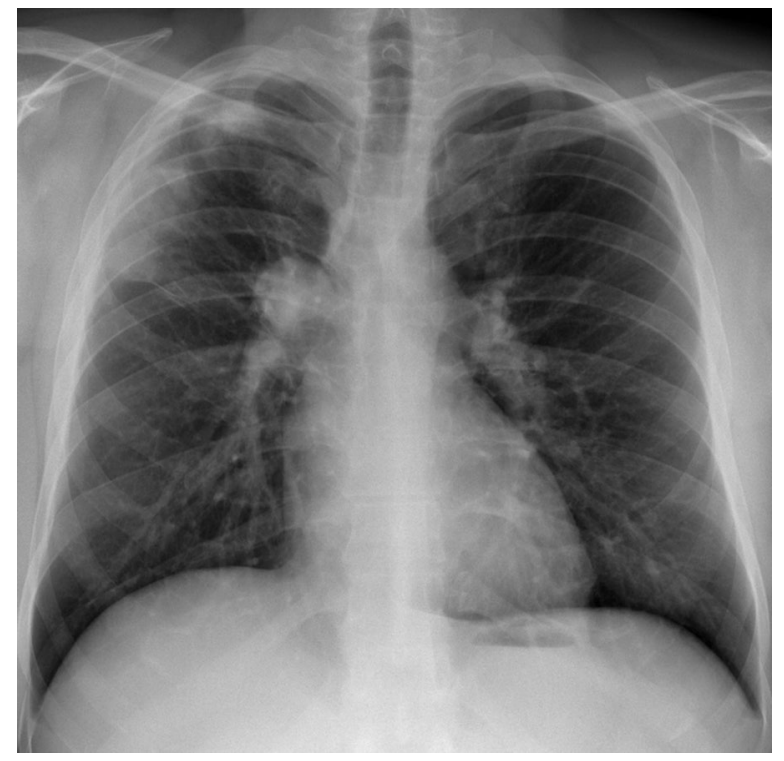

Figure 1. Chest $X$-ray: Patchy parenchymal densities, peripherally in the right upper lobe with right hilum enlargement caused by a round lesion with a diameter of $18 \mathrm{~mm}$

upper pole of the right hilum, a tumor, measuring $28 \times 22 \mathrm{~mm}$ was revealed - it was located within the right superior pulmonary vein, extending proximally in the direction of the left atrium and ending $10 \mathrm{~mm}$ from the outlet of the vein. The infiltration was extending distally in the dilated veins of segment 3, making up large tumour-like forms, measuring $30 \times 35 \mathrm{~mm}$. Within these visible lesions, there were thin vessels with marginal enhancement which could correspond to collateral circulation. Moreover, in segments 1 and 2 there were parenchymal densities, which could correspond to pulmonary infarction, a little smaller when compared to the examination made 10 days before (Figs. 2, 3). A hypothesis of the proliferative process was made, taking into consideration, first of all, angiosarcoma or leiomyosarcoma.

The location of the lesion within the lumen of the pulmonary veins with an extensive collateral circulation led to an idea to abandon the previously planned transthoracic fine-needle aspiration biopsy (TFNAB). After consulting a thoracic surgeon, the patient was qualified for surgery with intraoperative microscopic assessment of the surgical material. After two weeks of anticoagulation treatment, the patient underwent an antero-lateral thoracotomy with a resection of the fragment of lung parenchyma with a well-defined soft hyaline tumour, size: $3.4 \times 3.7 \mathrm{~cm}$, whitegreyish in colour with some satellite foci (Fig. 4). The intraoperative microscopic assessment of the tumour revealed a malignant tumour of the epithelial cells with necrosis. It was intraoperatively assessed that radical surgery was possible and thus a right upper bilobectomy was performed with intrapericardial ligation of the right superior pulmonary vein with lymphadenectomy.

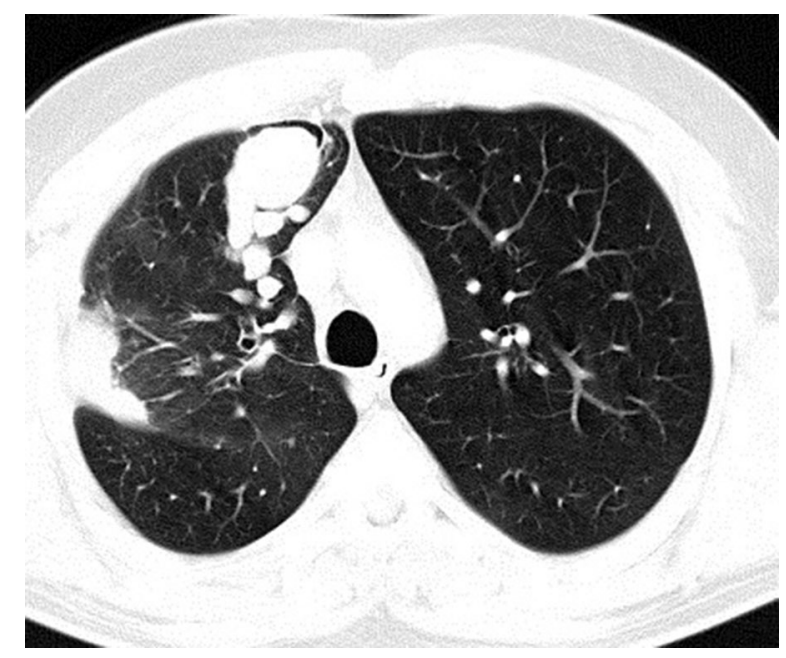

Figure 2. CT scan, pulmonary window: A polycyclic tumor and a few smaller nodules with smooth margins in the anterior segment of the right upper lobe and subpleural parenchymal density corresponding to a lung infarction in the posterior segment of this lobe

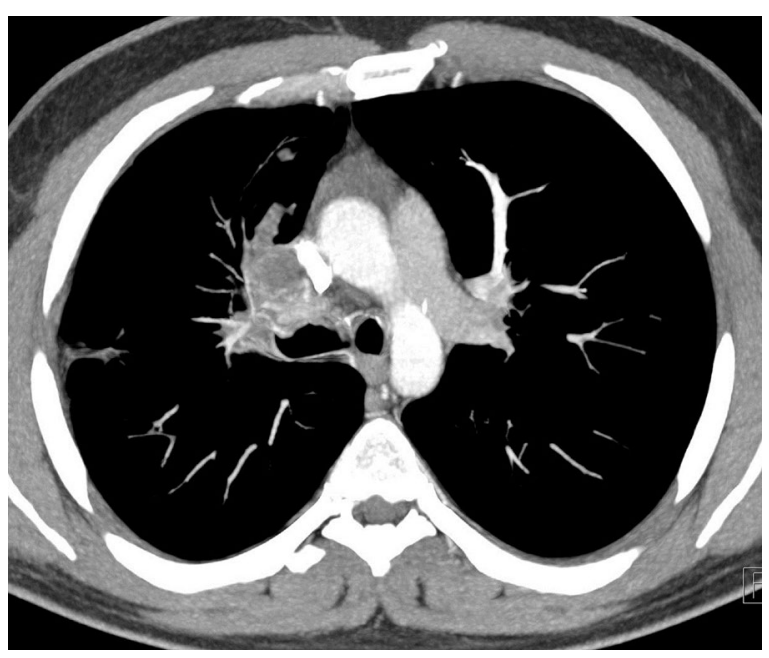

Figure 3. CT scan, mediastinal window, MIP reconstruction: tissue masses obliterating the dilated proximal segment of the right superior pulmonary vein, corresponding to an intravascular tumour mass. Small dilated collateral vessels within the hilum

Analysis of the post-operative specimen allowed an ultimate diagnosis of fetal adenocarcinoma with diffuse areas with a low (and focally high) degree of malignancy. Mucicarmine staining of the mucosal staining rendered negative result. In the immunohistochemistry tests, cytokeratin expression was found with a wide spectrum of (CKAE1/AE3), TTF-1, $\beta$-catenin, synaptophysin focally and $<10 \%$ CK7 cells, whilst chromogranin $A$ and vimentin were negative (Figs. 5, 6).

No tumor infiltration was found in the surgical margin of the bronchi and in the vessels and no metastases were found in the harvested fragments of the lymph nodes of the 11, 7 , 


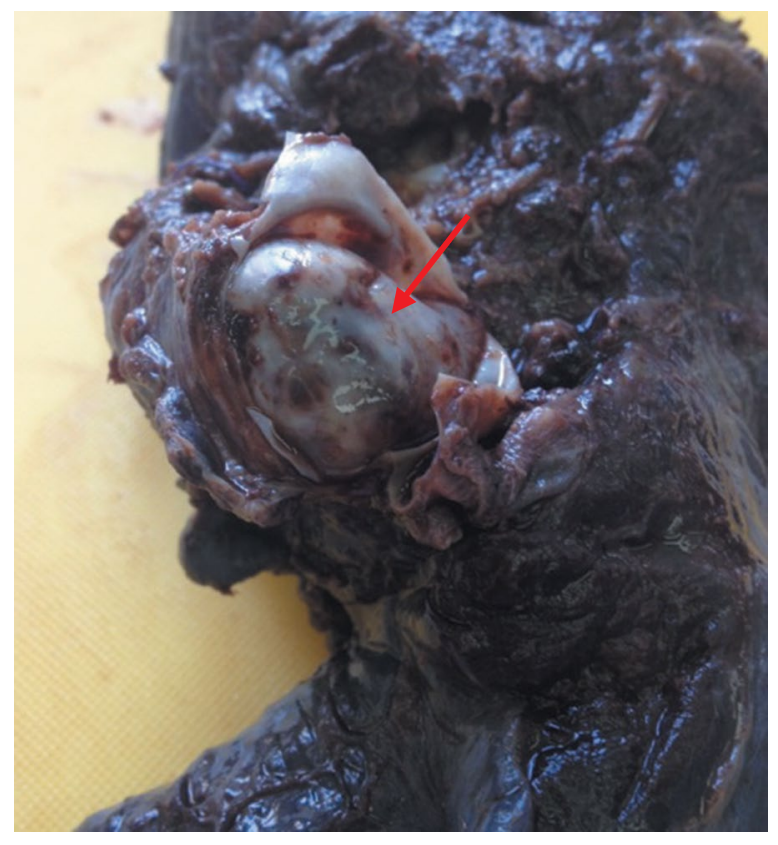

Figure 4. A lung with a visible tumour with a smooth, shiny surface, filling the lumen of a large branch of the pulmonary vein (red arrow)

4R and 10R group. The final diagnosis was adenocarcinoma fetale pulmonis dextri high-grade (pT3NOROLOV1) with IIB advancement stage. The postoperative course was uneventful.

Given the diagnosis of a fetal adenocarcinoma which usually has a good prognosis, and the radical type of surgery, which is the treatment of choice in this type of carcinoma as well as some equivocal recommendations concerning adjuvant treatment, the idea of adjuvant chemotherapy was abandoned. Nevertheless, after the discharge, the patient contacted another centre, where he received 4 cycles of chemotherapy based on cisplatin and etoposide. The patient remained under the supervision of an oncological and a thoracic-surgery clinic for 9 months. The follow-up chest computed tomography did not reveal any proliferative processes.

\section{Discussion}

The case of the presented patient shows that unusual complaints of the respiratory tract, paired with unremarkable results of blood laboratory tests should not be an argument against a thorough diagnostic process, being an indication at the very least for a chest X-ray. The described case encourages a broader look at the patient with adenocarcinoma. The diagnosis of fetal adenocarcinoma, in comparison with other lung adenocarcinomas, is usually connected with good prognosis, which, given the young average age of the subjects may have a significant influence factor on their planning of the future.

This paper describes a case of a 32-year old male patient with a cigarette smoking history, in whose case the results

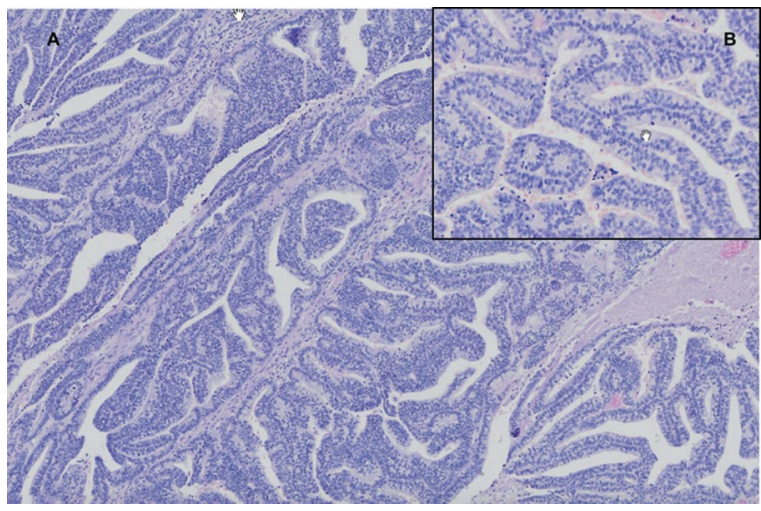

Figure. 5. Microscopic evaluation of lung tumour specimen. The architecture of fetal adenocarcinoma with irregular tubules (A), lined with glandular epithelium with clear cytoplasm (B). Small and large magnification, HE staining

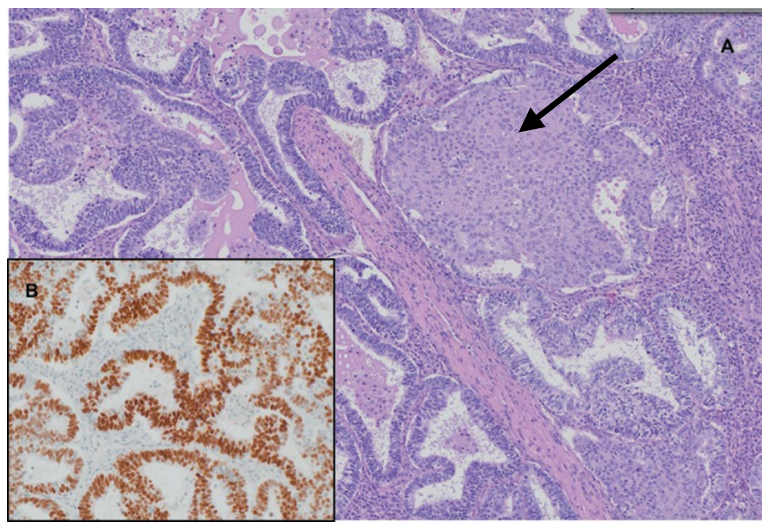

Figure 6. The fragments of fetal adenocarcinoma with the presence of morules (arrow). HE staining (A); Immunohistochemistry assay with TTF-1 antibody. A positive reaction within the cancer cells is visible. Large magnification (B)

of a thorough diagnostic process led to a diagnosis of fetal adenocarcinoma. The average age of patients, at the moment of diagnosis, is the $4^{\text {th }}$ decade of life, so the majority of patients are young people, prevalently cigarette smokers [9, $10,14]$. The disease frequently is asymptomatic, with tumourlike lesions in the lungs which are found accidentally during periodical medical check-ups $[2,9]$. The symptoms of fetal adenocarcinoma, once they occur, are not typical and comprise: dry cough, fever, debilitation and fatigue. The more worrying symptoms are haemoptysis and chest pain $[2,3,7,13$, 15-17]. One of the rare causes of chest pain is the presence of fluid in the pleural cavity caused by its involvement with cancer, or secondary lesions connected with emboli in the pulmonary vessels accompanying the adenocarcinoma $[15,18]$. The patient, whose case is discussed here, reported mainly a cough and chest pain. During outpatient treatment, he also experienced some haemoptysis. In his chest X-ray, the atelectasis of the right upper lobe was found with a smooth- 
-contoured tumor, measuring $44 \times 30 \mathrm{~mm}$. The abnormalities in the chest X-ray of such patients, which usually reveal atelectasis or a well-defined tumor, usually make up about $76 \%$ cases. The lesions tend to be situated in the right lung, and, in particular, similarly as in the case of the presented patient, in the upper lobe $[2,10,15]$. The chest computed tomography usually reveals a well-defined tumour mass with an average size of $3.5-4.5 \mathrm{~cm}$. The numerous nodular tumour-like lesions and intra-bronchial lesions are quite infrequent, and the involvement of the lymph nodes or the pleural cavity is rare $[2,10]$. In some patients, a chest computed tomography reveals, apart from oncological lesions, lesions corresponding to a pulmonary infarction [6]. The cause of these lesions might by the thromboembolism accompanying the cancer, or, as was the case here, such lesions were the result of the impotency of the pulmonary vessels obstructed by the tumour mass. The computed tomography of this patient's chest revealed a tumour of the right hilum, measuring $28 \times 22 \mathrm{~mm}$, located within the right superior pulmonary vein extending proximally towards the left atrium. The infiltration was extending distally in the dilated veins of segment 3 , making up large tumour-like forms, measuring $30 \times 35 \mathrm{~mm}$. Moreover, in segments 1 and 2, there were parenchymal densities, which could correspond to pulmonary infarction. No description of adenocarcinoma of the lung, involving pulmonary vessels, has been found in available publications.

In the discussed case the diagnosis was made on the basis of a intraoperative microscopic assessment. The location of the lesions and their unclear type led to giving up the idea of the previously planned CT-guided transthoracic fine-needle aspiration biopsy (TFNAB).

Preoperative assessment of the specimen collected with a bronchoalveolar brush or transthoracic fine needle aspiration biopsy (TFNAB) usually allows a hypothesis of a neuroendocrine tumour to be made. The final diagnosis, similarly as in this case, is possible only after a surgery and a microscopic assessment of the resected tumour.

The treatment of choice is surgery - the resection of the lobe with the tumour connected with the lymphadenectomy. In the discussed case, with regards to the location of the tumour and its extensity, a right upper bilobectomy was performed with intrapericardial ligation of the right superior pulmonary vein and with a mediastinal lymphadenectomy.

Fetal adenocarcinoma is still a rare disease, so the collection of a representative group of patients in order to define treatment standards remains impossible. Even though there are reports about the benefits in adjuvant chemotherapy and radiotherapy, these are not recommended treatments. The largest number of cases with equivocal results concern the administration of adjuvant chemotherapy containing cisplatin with etoposide $[1,16,17]$.

The prognosis of patients with fetal adenocarcinoma is usually good. The presented patient remained under the supervision of an oncological and a thoracic-surgery clinic for 9 months with follow-up chest computed tomography not revealing any proliferative processes.

The above article discussed a case of a rare variant of histological lung adenocarcinoma - a fetal adenocarcinoma. What makes this case quite unique is the fact that the changes were located within the pulmonary veins, which posed an additional diagnostic and treatment problem.

\section{Conflict of interest: none declared}

\section{Jolanta Winek, MD, PhD}

$10^{\text {th }}$ Department, Institute of Tuberculosis and Lung Diseases

Płocka 26, 01-138 Warszawa, Poland

e-mail:j.winek@igichp.edu.pl

Received: 19 Jun 2016

Accepted: 20 Oct 2016

\section{References}

1. Osakwe N, Tong J, Rodgers $\mathrm{W}$ et al. A rare case of fetal adenoracinoma of the lung. Ann Thorac Surg 2014; 98: 2217-2218.

2. Furuya K, Yasumori K, Takeo S et al. Well-differentiated fetal adenoracinoma of the lung: early-phase sequential high-resolution computed tomographic findings. J Comput Assist Tomogr 2008; 32: 806-809.

3. Sheehan KM, Curran J, Kay EW et al. Well differentiated fetal adenorcinoma of the lung in a 29 year old woman. JClin Pathol 2003;56:478-479.

4. Szołkowska M, Langfort R, Szczepulska-Wójcik E et al. Zmiany w klasyfikacji pierwotnego raka gruczołowego płuca przedstawione w rekomendacjach International Association for the Study of Lung Cancer/ American Society/ European Respiratory Society. Pneumonol Alergol Pol 2012; 80: 163-171.

5. Mardini G, Pai U, Chavez A et al. Endobronchial adenocarcinoma with endometrioid features and prominent neuroendocrine differentiation. A variant of fatal adenocarcinoma. Cancer 1994; 73: 1383-1389.

6. Fujino $S, A$ sada $Y$, Konishi $T$ et al. Well-differentiated fetal adenocarcinoma of lung. Lung Cancer 1995; 13; 311-316.

7. Longo $M$, Levra $M$, Capelletto E et al. Fetal adenocarcinoma of the lung in a 25-year-old woman. J Thorac Oncol 2008; 3: 441-443.

8. Suzuki M, Yazawa T, Ota $\mathrm{S}$ et al. High-grade fetal adenorcinoma of the lung is a tumour with a fetal phenotype that shows diverse differentiation, including high-grade neuroendocrine adenocarcinoma: a clinicopathological, immunihostochemical and mutational study of 20 cases. Histopathology 2015; 67: 806-816.

9. Geisinger K, Travis W, Perkins L et al. Aspiration cytomorphology of fetal adenoracinoma of the lung. Am J Clin Pathol 2010; 134: 894-902.

10. Sato $S$, Koike T, Yamato $Y$ et al. Resected well-differentiated fetal pulmonary adenocarcinoma and summary of 25 cases reported in Japan. Jpn J Thorac Cardiovasc Surg 2006; 54: 539-542.

11. Nakatani Y, Masudo K, Miyagi Y et al. Aberrant nuclear localization and gene mutation of $\beta$-catenin in low-grade adenocarcinoma of fetal lung type: up-regulation of the Wnt signalling pathway may be a common denominator for the development of tumors that form morules. Mod Pathol 2002; 15: 617-624.

12. de Kock L, Bah I, Wu Y et al. Germline and somatic DICER1 Mutations in a well- differentiated fetal adenocarcinoma of the lung. J Thorac Oncol 2016: 11: e31-e33.

13. Patnayak R, Jena $A$, Rukmangadha $\mathrm{N}$ et al. Well-differentiated fetal adenocarcinoma of the lung in an adult male: report of an unusual tumor with a brief review of literature. J Cancer Res Ther 2014; 10: 419-421.

14. Kadota K, Haba R, Katsuki N et al. Bronchial brushing cytology of a pulmonary fetal adenorcinoma with a poorly differentiated component. Cytopathology 2010; 21:349-351.

15. Thompson R, Hasleton $\mathrm{P}$, Taylor $\mathrm{P}$ et al. Haemoptysis in pregnancy caused by a well-differentiated fetal adenoracinoma: a case report J Med Case Rep 2010; 4: 17.

16. Giusti R, lacono D, Ida P et al. Multidisciplinary approach to fetal adenocarcinoma of the lung: a case report. Thorac Cancer 2014; 5: 97-100.

17. Lkhoyaali S, Boutayeb S, Ismaili $\mathrm{N}$ et al. Neoadjuvant chemotherapy in well- differentiated fetal adenocarcinoma: a case report. BMC Res Notes 2014; 7: 283.

18. Kneafsey P, Duggan MA, McFadden S. Fine needle aspiration cytology of pulmonary, well-differentiated fetal adenocarcinoma prepared by the ThinPrep method. Cytopathology 2003; 14: 87-90. 